International Mathematical Forum, 1, 2006, no. 15, 713-722

\title{
Meromorphic Functions with Positive Coefficients
}

\author{
Osama Nasser and Maslina Darus \\ School of Mathematical Sciences \\ Faculty of Science and Technology \\ Universiti Kebangsaan Malaysia, Bangi 43600 Selangor D.E., Malaysia \\ maslina@pkrisc.cc.ukm.my
}

\begin{abstract}
In this paper we consider the class $L(\alpha, \beta, \gamma, A, B, p)$ consisting of analytic and multivalent functions of order $p$ with positive coefficients. The aim of the study is to present some results on coefficient estimates, convex linear combinations, some distortion theorems and radii of starlikeness and convexity for functions $f$ in the class $L(\alpha, \beta, \gamma, A, B, p)$.
\end{abstract}

Mathematics Subject Classification: Primary 30C45

Keywords: Analytic functions, meromorphic functions, positive coefficients, growth and distortion theorem.

\section{Introduction}

Let $\Sigma_{p}$ denotes the class of functions of the form

$$
f(z)=\frac{1}{z^{-p}}+\sum_{n=1}^{\infty} a_{p+n-1} z^{p+n-1} \quad(p \in N)
$$

which are normalized analytic $p$-valent in the punctured disk $E_{0}=\{z: 0<$ $|z|<1\}$. A function $f \in \Sigma p$ is said to be, the class of meromorphically starlike of order $\rho$ if it satisfies the following:

$$
\operatorname{Re}\left(\frac{-z f^{\prime}(z)}{f(z)}\right)>\rho, \quad(z \in E)
$$

for some $\rho(0 \leq \rho<p)$. All such functions are denoted by $\Sigma^{*}(p, \rho)$. Similarly a function $\mathrm{f}$ in $\sum_{p}$ is said to be meromorphically convex of order $\rho$, if it satisfies 
the following:

$$
\operatorname{Re}\left(-p-\frac{z f^{\prime \prime}(z)}{f^{\prime}(z)}\right)>\rho, \quad(z \in E)
$$

for some $\rho(0 \leq \rho<p)$. All such functions are denoted by $\Sigma_{c}(p, \rho)$. The function $\mathrm{f}$ is in the class $\Sigma_{c}(p, \rho)$ if and only if $-z f^{\prime}(z) \in \Sigma^{*}(p, \rho)$.

Many important properties and characteristics of various interesting subclasses of the class $\Sigma_{p}$ of meromorphic p-valent functions, including the classes $\Sigma_{p}^{*}(\rho)$ and $\Sigma_{p}^{C}(\rho)$ defined above, were studied rather extensively by (among others) Uralgeddi[1], Aouf et.al([4][5], Kulkarni et.al [8], Mogra([6],[7]) and Srivastava et.al $([2],[3])$.

First we define the class of functions $L(\alpha, \beta, \gamma, A, B, p)$ as follows:

Definition 1.1. A function $f$ given by (1.1) is said to be a member of the class $L(\alpha, \beta, \gamma, A, B, p)$ if it satisfies

$$
\left|\frac{z^{p+1} f^{\prime}(z)+z^{p} f(z) p}{[(B-A) \gamma-A] z^{p+1} f^{\prime}(z)-z^{p} f(z)[(B-A) \gamma \alpha-A p]}\right| \leq \beta,
$$

where $0<\beta \leq 1,0 \leq \alpha<p,-1<A \leq B \leq 1$ and $\frac{A}{B-A} \leq \gamma \leq 1$ for all $z \in E$.

\section{Coefficient Inequalities}

The following theorem gives a sufficient condition for a function $f$, analytic in $E$ to be in $L(\alpha, \beta, \gamma, A, B, p)$.

Theorem 2.1 Let the function $f$ be defined by (1.1). If

$$
\begin{gathered}
\sum_{n=1}^{\infty}(2 p+n-1)+\beta(B-A) \gamma(p+n-1-\alpha)-\beta A(n-1) a_{p+n-1} \\
\leq \beta(B-A) \gamma(p+\alpha)-2 \beta A p,
\end{gathered}
$$

where $0<\beta \leq 1,0 \leq \alpha<p,-1<A \leq B \leq 1$, and $\frac{A}{B-A} \leq \gamma \leq 1$ for all $z \in E$, then $f \in L(\alpha, \beta, \gamma, A, B, p)$.

Proof. Let us suppose that

$$
\begin{aligned}
\sum_{n=2}^{\infty}(2 p+n-1)+ & \beta(B-A) \gamma(p+n-1-\alpha)-\beta A(n-1) a_{p+n-1} \\
\leq & \beta(B-A) \gamma(p+\alpha)-2 \beta A p
\end{aligned}
$$


for $f \in \Sigma_{p}$ ). It suffices to show that

$$
\left|\frac{z^{p+1} f^{\prime}(z)+z^{p} f(z) p}{[(B-A) \gamma-A] z^{p+1} f^{\prime}(z)-z^{p} f(z)[(B-A) \gamma \alpha-A p]}\right| \leq \beta,(z \in E)
$$

Now:

$$
\begin{aligned}
& \left|\frac{z^{p+1} f^{\prime}(z)+z^{p} f(z) p}{[(B-A) \gamma-A] z^{p+1} f^{\prime}(z)-z^{p} f(z)[(B-A) \gamma \alpha-A p]}\right|= \\
& \left|\frac{\sum_{n=1}^{\infty}(2 p+n-1) a_{p+n-1} z^{2 p+n-1}}{-(B-A) \gamma(p+\alpha)+2 A p+\sum_{n=1}^{\infty}(B-A) \gamma(p+n-1-\alpha)-A(n-1) a_{p+n-1} z^{2 p+n-1}}\right|, \\
& \leq \frac{\sum_{n=1}^{\infty}(2 p+n-1)\left|a_{p+n-1}\right|}{(B-A) \gamma(p+\alpha)-2 A p-\sum_{n=1}^{\infty}(B-A) \gamma(p+n-1-\alpha)-A(n-1)\left|a_{p+n-1}\right|}<\beta .
\end{aligned}
$$

Then

$$
\begin{aligned}
\sum_{n=1}^{\infty}(2 p+n-1)+ & \beta(B-A) \gamma(p+n-1-\alpha)-\beta A(n-1) a_{p+n-1} \\
\leq & \beta(B-A) \gamma(p+\alpha)-2 \beta A p .
\end{aligned}
$$

When $n=1$, we have

$$
a_{p} \leq \frac{\beta(B-A) \gamma(p+\alpha)-2 \beta A p}{2 p+\beta(B-A) \gamma(p-\alpha)}
$$

This is equivalent to our condition of the theorem, so that $f \in L(\alpha, \beta, \gamma, A, B, p)$.

The result is attained for a function $f$ given by

$$
\begin{gathered}
f(z)=z^{-p}+\frac{\beta(B-A) \gamma(p+\alpha)-2 \beta A p}{(2 p+n-1)+\beta(B-A) \gamma(p+n-1-\alpha)-\beta A(n-1)} z^{p+n-1}, \\
(n \geq 1)
\end{gathered}
$$

Corollary 2.1. Let the function $f$ be defined by (1.1). If $f \in L(\alpha, \beta, \gamma, A, B, p)$, then

$$
a_{p+n-1} \leq \frac{\beta(B-A) \gamma(p+\alpha)-2 \beta A p}{(2 p+n-1)+\beta(B-A) \gamma(p+n-1-\alpha)-\beta A(n-1)},(n \geq 1)
$$

The result is sharp for functions $f$ given by (2.4). 


\section{Distortion Theorem}

A distortion property for functions $f$ to be in the class $L(\alpha, \beta, \gamma, A, B, p)$, is given as follows:

Theorem 3.1. If the function $f$ be defined by (1.1) is in the class

$L(\alpha, \beta, \gamma, A, B, p)$, then for $0<|z|=r<1$, we have

$$
\begin{gathered}
\frac{1}{r^{p}}-\frac{\beta(B-A) \gamma(p+\alpha)-2 \beta A p}{2 p+\beta(B-A) \gamma(p-\alpha)} r^{p} \\
\leq|f(z)| \leq \frac{1}{r^{p}}+\frac{\beta(B-A) \gamma(p+\alpha)-2 \beta A p}{2 p+\beta(B-A) \gamma(p-\alpha)} r^{p}
\end{gathered}
$$

with equality holds for

$$
f(z)=\frac{1}{z^{p}}+\frac{\beta(B-A) \gamma(p+\alpha)-2 \beta A p}{2 p+\beta(B-A) \gamma(p-\alpha)} z^{p}, \quad(z=i r, r)
$$

and

$$
\begin{gathered}
\frac{p}{r^{p+1}}-\frac{p[\beta(B-A) \gamma(p+\alpha)-2 \beta A p]}{2 p+\beta(B-A) \gamma(p-\alpha)} r^{p-1} \\
\leq\left|f^{\prime}(z)\right| \leq \frac{p}{r^{p+1}}+\frac{p[\beta(B-A) \gamma(p+\alpha)-2 \beta A p}{2 p+\beta(B-A) \gamma(p-\alpha)} r^{p-1},
\end{gathered}
$$

with equality holds for function $f$ given by

$$
f(z)=\frac{1}{z^{p-1}}+\frac{\beta(B-A) \gamma(p+\alpha)-2 \beta A B}{2 p+\beta(B-A) \gamma(p-\alpha)} z^{p-1}, \quad(z= \pm i r, \pm r) .
$$

Proof. Since $f \in L(\alpha, \beta, \gamma, A, B, p)$, Theorem 2.1 yields

$$
\sum_{n=1}^{\infty} a_{p+n-1} \leq \frac{\beta(B-A) \gamma(p+\alpha)-2 \beta A p}{(p+n-1)+\beta(B-A) \gamma(p+n-1-\alpha)-\beta A(n-1)},(n \geq 1)
$$

Thus, for $0<|z|=r<1$, and making use of (2.3) we have

$$
\begin{aligned}
|f(z)| & \leq|z|^{-p}+\sum_{n=1}^{\infty} a_{p+n-1}|z|^{p+n-1} \\
& \leq r^{-p}+\frac{\beta(B-A) \gamma(p+\alpha)-2 \beta A p}{(2 p)+\beta(B-A) \gamma(p-\alpha)} r^{p}
\end{aligned}
$$


and

$$
\begin{aligned}
|f(z)| & \geq|z|^{-p}-\sum_{n=1}^{\infty} a_{p+n-1}|z|^{p+n-1} \\
& \geq r^{-p}-\frac{\beta(B-A) \gamma(p+\alpha)-2 \beta A p}{(2 p)+\beta(B-A) \gamma(p-\alpha)} r^{p} .
\end{aligned}
$$

Also from Theorem 2.1, it follows that

$$
\sum_{n=1}^{\infty} a_{p+n-1} \leq \frac{\beta(B-A) \gamma(p+\alpha)-2 \beta A p}{(2 p)+\beta(B-A) \gamma(p-\alpha)}
$$

and hence

$$
\begin{aligned}
\left|f^{\prime}(z)\right| & \leq p|z|^{-p-1}+\sum_{n=1}^{\infty}(p+n-1) a_{p+n-1}|z|^{p+n-2} \\
& \leq p r^{-p-1}+\frac{p[\beta(B-A) \gamma(p+\alpha)-2 \beta A p]}{2 p+\beta(B-A) \gamma(P-\alpha)} r^{p-1}
\end{aligned}
$$

Thus

$$
\begin{aligned}
\left|f^{\prime}(z)\right| & \geq p|z|^{-p-1}-\sum_{n=1}^{\infty}(p+n-1) a_{p+n-1}|z|^{p+n-2} \\
& \geq p r^{-p-1}-\frac{p[\beta(B-A) \gamma(P+\alpha)-2 \beta A p]}{2 p+\beta(B-A) \gamma(P-\alpha)} r^{p-1} .
\end{aligned}
$$

Hence completes the proof of Theorem 3.1

\section{Radii of Starlikeness and Convexity}

The radii of starlikeness and convexity for the class $L(\alpha, \beta, \gamma, A, B, p)$, is given by the following theorem:

Theorem 4.1. If the function $f$ be defined by (1.1) is in the class $L(\alpha, \beta, \gamma, A$, $B, p)$, then $f$ is starlike of order $\rho(0 \leq \rho<p)$, in the unit disk $|z|<$ $r_{1}(\alpha, \beta, \gamma, A, B, p)$, where $r_{1}(\alpha, \beta, \lambda, p, \rho)$, is the largest value for which $r_{1}(\alpha, \beta, \gamma, A, B, p)=$

$$
r_{1}=\inf _{n \geq 1}\left(\frac{(p-\rho)[(2 p+n-1)+\beta(B-A) \gamma(p+n-1-\alpha)-\beta A(n-1)]}{(\beta(B-A) \gamma(p+\alpha)-2 \beta A p)(p+n+1-\rho)}\right)^{\frac{1}{2 p+n-1}}
$$


The result is sharp for functions $f$ given by (2.4).

Proof. It suffices to show that

$$
\left|\frac{z f^{\prime}(z)}{f(z)}+1\right| \leq(1-\rho)
$$

for $|z| \leq r_{1}$, we have

$$
\begin{gathered}
\left|\frac{z f^{\prime}(z)}{f(z)}+1\right|=\left|\frac{\left[z^{-p}(-p+1)\right]+\sum_{n=1}^{\infty}(p+n) a_{p+n-1} z^{2 p+n-1}}{z^{-p}\left(1+\sum_{n=1}^{\infty} a_{p+n-1} z^{2 p+n-1}\right)}\right| \\
\leq \frac{(-p+1)+\sum_{n=1}^{\infty}(p+n) \frac{\beta(B-A) \gamma(p+\alpha)-2 \beta A p}{(2 p+n-1)+\beta(B-A) \gamma(p+n-1-\alpha)-\beta A(n-1)}|z|^{2 p+n-1}}{1-\sum_{n=1}^{\infty} \frac{\beta(B-A) \gamma(p+\alpha)-2 \beta A p}{(2 p+n-1)+\beta(B-A) \gamma(p+n-1-\alpha)-\beta A(n-1)}|z|^{2 p+n-1}} \\
\leq(1-\rho) .
\end{gathered}
$$

Hence (4.8) holds true if

$$
\sum_{n=1}^{\infty} \frac{(p+n+1-\rho)[\beta(B-A) \gamma(p+\alpha)-2 \beta A p]}{(2 p+n-1)+\beta(B-A) \gamma(p+n-1-\alpha)-\beta A(n-1)}|z|^{2 p+n-1} \leq(p-\rho),
$$

And it follows that

$$
\begin{gathered}
|z| \leq\left(\frac{(p-\rho)[(2 p+n-1)+\beta(B-A) \gamma(p+n-1-\alpha)-\beta A(n-1)]}{(p+n+1-\rho)[\beta(B-A) \gamma(p+\alpha)-2 \beta A p]}\right)^{\frac{1}{2 p+n-1}} \\
(n \geq 1)
\end{gathered}
$$

then,

$$
r_{1}=\inf _{n \geq 1}\left(\frac{(p-\rho)[(2 p+n-1)+\beta(B-A) \gamma(p+n-1-\alpha)-\beta A(n-1)]}{(\beta(B-A) \gamma(p+\alpha)-2 \beta A p)(p+n+1-\rho)}\right)^{\frac{1}{2 p+n-1}}
$$

as required.

Theorem 4.2. If the function $f$ defined by (1.1) is in the class $L(\alpha, \beta, \gamma, A, B, p)$, then $f$ is convex of order $\rho(0 \leq \rho<p)$, in the unit disk $|z|<r_{2}(\alpha, \beta, \gamma, A, B, p)$, where $r_{2}(\alpha, \beta, \gamma, A, B, p)$, is the largest value for which 
$r_{2}=\inf _{n \geq 1}\left(\frac{p(p-\rho)[(2 p+n-1)+\beta(B-A) \gamma(p+n-1-\alpha)-\beta A(n-1)]}{(p+n-1)(p+n+1-\rho)(\beta(B-A) \gamma(p+\alpha)-2 \beta A p)}\right)^{\frac{1}{2 p+n-1}}$.

The result is sharp for functions given by (2.4).

Proof. It suffices to show that

$$
\left|\frac{z f^{\prime \prime}(z)}{f^{\prime}(z)}+2\right| \leq(1-\rho)
$$

For $|z| \leq r_{2}$, we have

$$
\begin{aligned}
& \left|\frac{z f^{\prime \prime}(z)}{f^{\prime}(z)}+2\right|=\left|\frac{-\left(p-p^{2}\right) \sum_{n=1}^{\infty}(p+n)(p+n-1) a_{p+n-1} z^{2 p+n-1}}{-p+\sum_{n=1}^{\infty}(p+n-1) a_{p+n-1} z^{2 p+n-1}}\right| \\
& \leq \frac{\left(p-p^{2}\right)+\sum_{n=1}^{\infty} \frac{(p+n)(p+n-1) \beta(B-A) \gamma(p+\alpha)-2 \beta A p}{(2 p+n-1)+\beta(B-A) \gamma(p+n-1-\alpha)-\beta A(n-1)}|z|^{2 p+n-1}}{p-\sum_{n=1}^{\infty} \frac{(p+n-1)[\beta(B-A) \gamma(p+\alpha)-2 \beta A p]}{(2 p+n-1)+\beta(B-A) \gamma(p+n-1-\alpha)-\beta A(n-1)}|z|^{2 p+n-1}} \leq(1-\rho),
\end{aligned}
$$

Hence (4.9) holds true if

$$
\sum_{n=1}^{\infty} \frac{(p+n+1-\rho)(p+n-1)[\beta(B-A) \gamma(p+\alpha)-2 \beta A p]}{(2 p+n-1)+\beta(B-A) \gamma(p+n-1-\alpha)-\beta A(n-1)}|z|^{2 p+n-1} \leq p(p-\rho),
$$

And it follows that

$$
\begin{gathered}
|z| \leq\left(\frac{p(p-\rho)[(2 p+n-1)+\beta(B-A) \gamma(p+n-1-\alpha)-\beta A(n-1)]}{(p+n+1-\rho)(p+n-1)[\beta(B-A) \gamma(p+\alpha)-2 \beta A p]}\right)^{\frac{1}{2 p+n-1}}, \\
(n \geq 1) .
\end{gathered}
$$

Then

$$
r_{2}=\inf _{n \geq 1}\left(\frac{p(p-\rho)[(2 p+n-1)+\beta(B-A) \gamma(p+n-1-\alpha)-\beta A(n-1)]}{(p+n-1)(p+n+1-\rho)(\beta(B-A) \gamma(p+\alpha)-2 \beta A p)}\right)^{\frac{1}{2 p+n-1}}
$$

as required. 


\section{Convex linear Combination}

Our next result involves a linear combination of function of the type (1.1).

Theorem 5.1. Let

$$
f_{1}=z^{-p}+\frac{\beta(B-A) \gamma(p+\alpha)-2 \beta A p}{2 p+\beta(B-A) \gamma(p-\alpha)} z^{p}
$$

and

$$
\begin{gathered}
f_{n}(z)=z^{-p}+\frac{\beta(B-A) \gamma(p+\alpha)-2 \beta A p}{(2 p+n-1)+\beta(B-A) \gamma(p+n-1-\alpha)-\beta A(n-1)} z^{p+n-1}, \\
(n \geq 1),
\end{gathered}
$$

then $f \in L(\alpha, \beta, \gamma, A, B, p)$ if and only if it can be expressed in the form

$$
\begin{aligned}
& f(z)=\sum_{n=1}^{\infty} \lambda_{n} f_{n}(z), \\
& \text { where } \lambda_{n} \geq 0 \quad \text { and } \quad \sum_{n=1}^{\infty} \lambda_{n}=1
\end{aligned}
$$

Proof. $\Leftarrow$ From $(5.10),(5.11)$ and $(5.12)$, it is easily seen that

$$
\begin{gathered}
f(z)=\sum_{n=1}^{\infty} \lambda_{n} f_{n}(z) \\
=z^{-p}+\sum_{n=1}^{\infty} \frac{[\beta(B-A) \gamma(p+\alpha)-2 \beta A p] \lambda_{n}}{(2 p+n-1)+\beta(B-A) \gamma(p+n-1-\alpha)-\beta A(n-1)} z^{p+n-1} .
\end{gathered}
$$

Since

$$
\sum_{n=1}^{\infty} \frac{[\beta(B-A) \gamma(p+\alpha)-2 \beta A p] \lambda_{n}}{(2 p+n-1)+\beta(B-A) \gamma(p+n-1-\alpha)-\beta A(n-1)}=\sum_{n=1}^{\infty} \lambda_{n} \leq 1,
$$

it follows from Theorem 2.1 that the function $f \in L(\alpha, \beta, \gamma, A, B, p)$.

$\Leftarrow$ Conversely, let us suppose that $f \in L(\alpha, \beta, \gamma, A, B, p)$. Since

$$
a_{p+n-1} \leq \frac{\beta(B-A) \gamma(p+\alpha)-2 \beta A p}{(2 p+n-1)+\beta(B-A) \gamma(p+n-1-\alpha)-\beta A(n-1)} \quad(n \geq 1)
$$


Setting

$$
\lambda_{n}=\frac{(2 p+n-1)+\beta(B-A) \gamma(p+n-1-\alpha)-\beta A(n-1))}{\beta(B-A) \gamma(p+\alpha)-2 \beta A p} a_{p+n-1} \quad(n \geq 1)
$$

it follows that

$$
f(z)=\sum_{n=1}^{\infty} \lambda_{n} f_{n}(z)
$$

this complete the proof of theorem. And, finally we prove

Theorem 5.2. The class $L(\alpha, \beta, \gamma, A, B, p)$ is closed under convex linear combinations.

Proof. Suppose that the functions $f_{1}$ and $f_{2}$ defined by,

$$
f_{i}(z)=z^{-p}+\sum_{n=1}^{\infty} a_{p+n-1, i} z^{p+n-1} \quad(i=1,2 ; z \in E)
$$

be in the class $L(\alpha, \beta, \gamma, A, B, p)$.

Setting $\quad f(z)=\mu f_{1}(z)+(1-\mu) f_{2}(z) \quad(0 \leq \mu \leq 1)$,

from (5.13)we obtain

$$
f(z)=z^{-p}+\sum_{n=1}^{\infty}\left\{\mu a_{p+n-1,1}+(1-\mu) a_{p+n-1,2}\right\} z^{p+n-1}, \quad(z \in E)
$$

In view of theorem 2.1, we have

$$
\begin{gathered}
\sum_{n=1}^{\infty}[(2 p+n-1)+\beta(B-A) \gamma(p+n-1-\alpha)-\beta A(n-1)]\left\{\mu a_{p+n-1,1}+(1-\mu) a_{p+n-1,2}\right\} \\
\quad=\mu \sum_{n=1}^{\infty}[(2 p+n-1)+\beta(B-A) \gamma(p+n-1-\alpha)-\beta A(n-1)] a_{p+n-1,1} \\
+(1-\mu) \sum_{n=1}^{\infty}[(2 p+n-1)+\beta(B-A) \gamma(p+n-1-\alpha)-\beta A(n-1)] a_{p+n-1,2} \\
\leq \mu[\beta(B-A) \gamma(p+\alpha)-2 \beta A p]+(1-\mu)[\beta(B-A) \gamma(p+\alpha)-2 \beta A p] \\
=\beta(B-A) \gamma(p+\alpha)-2 \beta A p .
\end{gathered}
$$


which show that $f \in L(\alpha, \beta, \gamma, A, B, p)$.

ACKNOWLEDGEMENT: The work presented here was supported by IRPA 09-02-02-10029 EAR.

\section{References}

[1] B. A, Uralegaddi and M.D Ganigi, Meromorphic multivalent functions with positive coefficient, Nepali Math. Sci., Rep. 11, (1986), 95-102.

[2] H. M. Srivastava, H. M. Hossen and M. K. Aouf. A unified presentation of some classes meromorphically multivalent functions, Comp. Math. with App. 38, (1999), 63-70.

[3] H. M. Srivastava, M. K. Aouf. Some applications of fractional calculus operators to certain subclasses of prestarlike functions with negative coefficients, Comp. Math. with App. 30,(1995), 53-61.

[4] M. K. Aouf. On a class meromorphic multivalent functions with positive coefficients, Math. Japan. 35, (1990), 603-608.

[5] M. K. Aouf. A generalization of meromorphic multivalent functions with positive coefficients, Math. Japan. 35, (1990), 609-614.

[6] M. L. Mogra. Meromorphic multivalent functions with positive coefficients I, Math. Japan. 35, (1990), 1-11.

[7] M. L. Mogra. Meromorphic multivalent functions with positive coefficients II, Math. Japan. 35, (1990), 1089-1098.

[8] S. R. Kulkarni, U. H. Naik and H. M. Srivastava. A certain class of meromorphically p-valent, quasi-convex functions, Pan Amer. Math. J. 8 (1), (1998), 57-64.

Received: May 12, 2005 